\title{
Calculating the purchasing power of tourists in a wine region - a case study from Germany / Kaufkraft von Touristen in einer Weinregion - eine Fallstudie aus Deutschland
}

\author{
Gergely Szolnoki \\ Geisenheim University, Institut für Betriebswirtschaft und Marktforschung, Von-Lade-Str. 1, 65366 Geisenheim, Germany
}

\begin{abstract}
The main purpose of this consumer study was firstly to calculate the economic importance, in term of purchasing power, of visitors in the German wine growing region Rheingau, and secondly to examine their demographic and behavioural characteristics, as well as their the motivation using segmentation approach. In the framework of a face-to-face survey, conducted between April and May 2015, a total of 1,555 tourists were interviewed at 18 different locations in the Rheingau. Four wine tourist types were identified by using cluster analysis: 1) Wine and Rheingau lovers, who have a high interest in wine and visit the region several times a year; 2) wine-oriented tourists, who use the culinary offerings of the Rheingau with pleasure but do not come to the region very often; 3 ) new visitors, who come to the region mainly because of the nature and history; and 4) foreign tourists, who have less knowledge about the Rheingau and buy less wine in the region. With the help of the purchasing power calculation, we can state that approximately $18 \%$ of the total production is purchased directly in the region by tourists.
\end{abstract}

\begin{abstract}
Zusammenfassung: Ziel dieser Studie war es, zum einen die wirtschaftliche Bedeutung von Besuchern eines deutschen Anbaugebietes - Rheingau - in Form von Kaufkraft zu errechnen und zum anderen die demographischen bzw. verhaltensbezogenen Merkmale sowie die Motivation der Touristen mithilfe eines Segmentierungsansatzes zu analysieren. Im Rahmen einer face-to-face Befragung im April-Mai 2015 wurden insgesamt 1.555 Touristen an 18 Standorten im Rheingau interviewt. Mithilfe einer Cluster-Analyse konnten insgesamt vier unterschiedliche Besuchertypen identifiziert werden: 1) Wein- \& Rheingau-Liebhaber, die regelmäßig den Rheingau besuchen und hohes Interesse an Wein haben; 2) Weinaffine-Touristen, die gerne das kulinarische Angebot des Rheingaus in Anspruch nehmen, allerdings weniger regelmäßig die Region besuchen; 3) Neubesucher, die hauptsächlich wegen Natur und Tradition die Region besuchen; und 4) Ausländische Touristen, die am wenigsten Weinwissen aufweisen und am wenigsten Wein im Rheingau kaufen. Bei der Modellierung der Kaufkraft hat sich herausgestellt, dass ca. 18\% der Gesamtproduktion in der Region vor Ort von Touristen gekauft wird.
\end{abstract}

\section{Einleitung und methode}

Die Symbiose von Wein \& Tourismus im Rheingau ist ein Phänomen, das sich nur mit wenigen anderen Weinregionen der Welt vergleichen lässt. Das war der Anlass einer größeren regionalen Studie, im Rahmen derer rund 60 Studierende die Verbindung von Tourismus und Wein in der Region aus der Perspektive der Touristen und der Produzenten untersuchten.

Vom Mitte März bis Mitte Mai 2015 wurden an 18 Standorten insgesamt 1.555 Touristen zu ihrer Meinung über den Rheingau, dessen Angebot und natürlich zu seinem Wein befragt.

Als Befragungsstandorte für die Besucher wurden touristisch relevante Punkte im mittleren Rheingau mit unterschiedlicher Frequentierung in Assmannshausen, Rüdesheim, Geisenheim, Oestrich-Winkel, Hattenheim und Eltville ausgewählt. Die Befragungen wurden sowohl in deutscher als auch in englischer Sprache durchgeführt. Ziel des Projektes war es zu erfahren, woher und aus welchen Gründen Menschen den Rheingau besuchen, $\mathrm{zu}$ welchen Anlässen sie kommen und wie lange sie bleiben. Es wurde versucht zu eruieren, inwiefern der Rheingau als Weinbauregion wahrgenommen wird und wie stark der Tourismus mit dem Ab-Hof-Verkauf der Winzer zusammenhängt. Des Weiteren ergab sich ein umfassendes Bild von der Meinung des durchschnittlichen Rheingautouristen, die Häufigkeit seiner Besuche, seinen Freizeitaktivitäten während des Urlaubs und der gewählten Unterkunft. Letztendlich strebte das Projekt neben der detaillierten Analyse der Besucher an, Schwächen der Region zu erkennen und Lösungsvorschläge oder Handlungsalternativen aufzuzeigen, um die Popularität des Rheingaus weiter zu steigern sowie Tourismus und Weinverkauf anzukurbeln.

\section{Literaturüberblick}

In den letzten Jahrzehnten ist eine große Anzahl von Artikeln über Weintourismus veröffentlicht worden. Carlsen und Charters (2006), Grybovych et al. (2013) sowie Molina et al. (2015) haben in ihren Papers eine gute Zusammenfassung der bisher publizierten Studien, deswegen verzichten wir in diesem Kapitel auf eine 
Table 1. Überblick der Weintourismus-Studien mit Segmentierungsansatz.

\begin{tabular}{lll}
\hline Autor & Lund & Segmentationsansatz \\
\hline Hall und Macionis (1998) & Australien, Neuseeland & Interesse \\
Charters und Ali-Knight (2000** & Australien & Interesse und Wissen \\
Bruwer (2003)* & Südafrika & Interesse \\
Brown et al. (2006) & Kanada & Interesse \\
Cullen et al. (2006) & Kanada & Interesse \\
Houghton (2008)* & Australien & Interesse \\
Gatti und Maroni (2004) & Italien & Motivation \\
Piscitelli et al. (2005) & Italien & Motivation \\
Bitsani und Kavoura (2012) & Italien & Motivation \\
Marzo-Navarro und & Spanien & Interesse und Wissen \\
Pedraja-Iglesias (2009) & Spanien & Interesse und Wissen \\
Nella und Christoui (2014) & Griechenland & Involvement \\
Di-Gregorio und Licari (2006) & Italien & Interesse \\
Molina et al. (2015) & Spanien & Interesse und Wissen \\
Alebaki und Iakovidou (2011) & Griechenland & Interesse und Motivation \\
Williams und Dossa (2003) & Kanada & Eistellung und Verhalten \\
Simpson und Bretherton (2004) & Neuseeland & Lifestyle \\
Chen und Sasias (2014) & Taiwan & Kulturelle Werten und Lifestyle \\
Galloway et al. (2008) & Australien & Sensation-suchendes Verhalten \\
Cho et al. (2014) & USA & Psychografische Eigenschaften \\
Dodd und Bigotte (1997) & USA/Texas & Alter und Einkommen \\
Tassiopoulos et al. (2004) & Südafrika & Besuchs-häufigkeit \\
Alonso et al. (2007) & Neuseeland & Nationalität \\
\hline
\end{tabular}

detaillierte Beschreibung von bereits existierenden Studien und geben eher eine kurze Fazit von Arbeiten, welche Weintouristen segmentiert haben.

Ziel dieses Kapitels ist es, einen Überblick über die unterschiedlichen Segmentierungsansätze, die andere Autoren bis jetzt verwendet haben, zu geben (Table 1).

Tabelle 1 macht es deutlich, dass die meisten Studien Interesse und Motivation als Segmentierungskriterium verwendet haben.

\section{Ergebnisse}

\subsection{Deskriptive beschreibung der besucher}

Ein Viertel der Befragten sind Erstbesucher im Rheingau, die die Region weiterempfehlen und so durch Mundpropaganda den potentiellen Besucherkreis erweitern. Weitere $30 \%$ gaben an, den Rheingau ein bis vier Mal pro Jahr zu besuchen. Die meisten Touristen reisten dabei mit dem Auto an, allerdings übernachtete nur knapp ein Drittel im Rheingau, bevorzugt in Hotels.

Das Veranstaltungsangebot spielt im Rheingau eine wichtige Rolle. Am beliebtesten waren die Schlemmerwoche, Rhein in Flammen und der Rüdesheimer Weihnachtsmarkt. Auf die Frage hin, wie groß die Zufriedenheit mit dem Freizeitangebot sei, war die Resonanz durchweg positiv. Vor allem das Weinangebot, die Gastronomie bzw. der Rheingau als Ausflugsziel wurden gelobt. Wie auch aus Abb.1 ersichtlich, sahen die Besucher allerdings noch Defizite bei den Einkaufsmöglichkeiten und den Angeboten für Familien und Kinder. Bei der Frage nach Begriffsassoziationen mit dem Rheingau waren über zwei Drittel der Antworten weinbezogen, die anderen bezogen sich auf die Umgebung und die Natur des Rheingaus.

\subsection{Segmentierung der besucher}

Alonso et al. (2007) haben in ihrer Studie den Unterschied zwischen einheimischen und ausländischen Besuchern analysiert, während Tassiopoulos et al. (2004) bzw. Molina et al. (2015) untersuchten u.a. die Besuchshäufigkeit von Touristen. Basierend auf diese Ergebnisse wurde eine Segmentierung mit den Faktoren Besuchshäufigkeit, Nationalität und Anzahl der durchschnittlich gekauften Flaschen durchgeführt.

Es konnten insgesamt vier unterschiedliche Besuchertypen identifiziert werden: 1) Wein- \& RheingauLiebhaber gehören zu der Gruppe der Häufig-Trinker, besuchen regelmäßig den Rheingau und kaufen im Schnitt mehr als 16 Flaschen Wein im Rheingau; 2) auch weinaffine Rheingau-Touristen konsumieren überdurchschnittlich viel Wein, allerdings besuchen sie weniger regelmäßig die Region und kaufen weniger Wein; 3) Neubesucher konsumieren im Vergleich zu den ersten zwei Segmenten deutlich weniger Wein, sind zum ersten Mal oder seltener als einmal pro Jahr im Rheingau und kaufen durchschnittlich 6 Flaschen Wein in der Region; 4) Ausländische Touristen, von denen mehr als die Hälfte zum ersten Mal den Rheingau besuchen und die gar keinen oder sehr wenig Wein hier kaufen.

Auffällig war, dass die Wein- \& Rheingau-Liebhaber zur Hälfte aus Hessen kamen und jeder Zweite ein abgeschlossenes Studium hatte. Diese Gruppe bevorzugte trockene, deutsche Weißweine, am liebsten direkt vom Winzer und beteiligte sich am meisten an den Veranstaltungen, auch wenn viele nur Tagesbesucher waren. Dieses Segment fühlte sich mit am besten über anstehende Veranstaltungen informiert und gab als Reisegrund hauptsächlich Weinreise und Erholung an. Analysiert man die Zahl der gekauften Flaschen in der Region, bezog dieses Segment den meisten Wein. Auch besuchten 


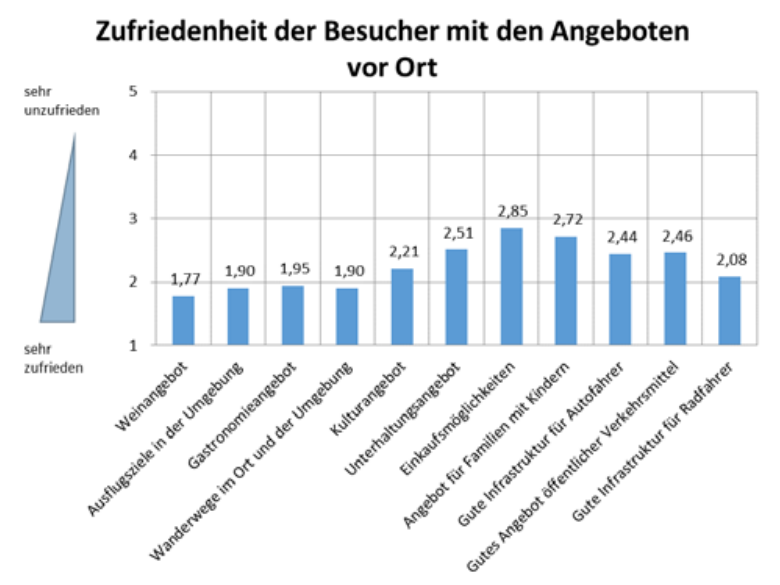

Abb 1. Analyse der Zufriedenheit der Besucher.

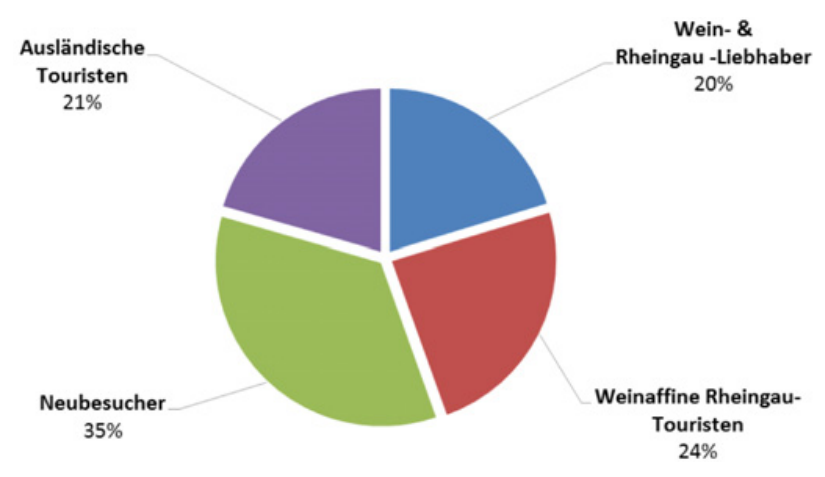

Abb 2. Besuchersegmente der Besucher.

sie die meisten Weingüter über die sie Informationen hauptsächlich aus dem Internet und über Mundpropaganda erhielten. Zusätzlich wurden das Weinangebot und die Weinqualität der Region im Gesamten von diesem Segment am besten bewertet.

Unter den weinaffinen Besucher waren vor allem Häufig- oder Gelegentlich-Trinker vertreten. Hier kam jeder Dritte aus Hessen und etwas mehr als zwei Drittel hat ein abgeschlossenes Studium. Bevorzugt wurden auch von ihnen trockene Weine direkt vom Winzer. Die verwendeten Bezugswege dieser Gruppe zeichnen sich durch eine signifikante Nutzung des Weinfachhandels aus. Dieses Segment bewertete das Angebot für Familien und Kinder mit am schlechtesten, auch die Infrastruktur für Autofahrer wurde kritisch angemerkt. Die am meisten besuchte Veranstaltung dieses Segments war das Rheingau Musikfestival.

Neubesucher kamen zu gut einem Fünftel aus Rheinland-Pfalz und zeichneten sich als Selten- bis Gelegentlich-Trinker aus. Diese Besuchergruppe nutzt am meisten das Freizeitangebot des Rheingaus in Form von Radurlauben, Wanderungen bzw. Schifffahrten, bewertet allerdings das Kulturangebot im Schnitt schlechter. Auch die Infrastruktur für Autos und der Informationsfluss wurden von dieser Gruppe kritisiert.

Im letzten Segment - ausländische Touristen - war der größte Anteil der Nicht- und Selten-Trinker zu finden, welche, falls sie Wein konsumierten, internationale Weine bevorzugten. Diese Besucher bleiben oft länger, kommen verstärkt mit Reisebussen oder Schiffen und nutzen am häufigsten die Touristeninformation. Auch bei

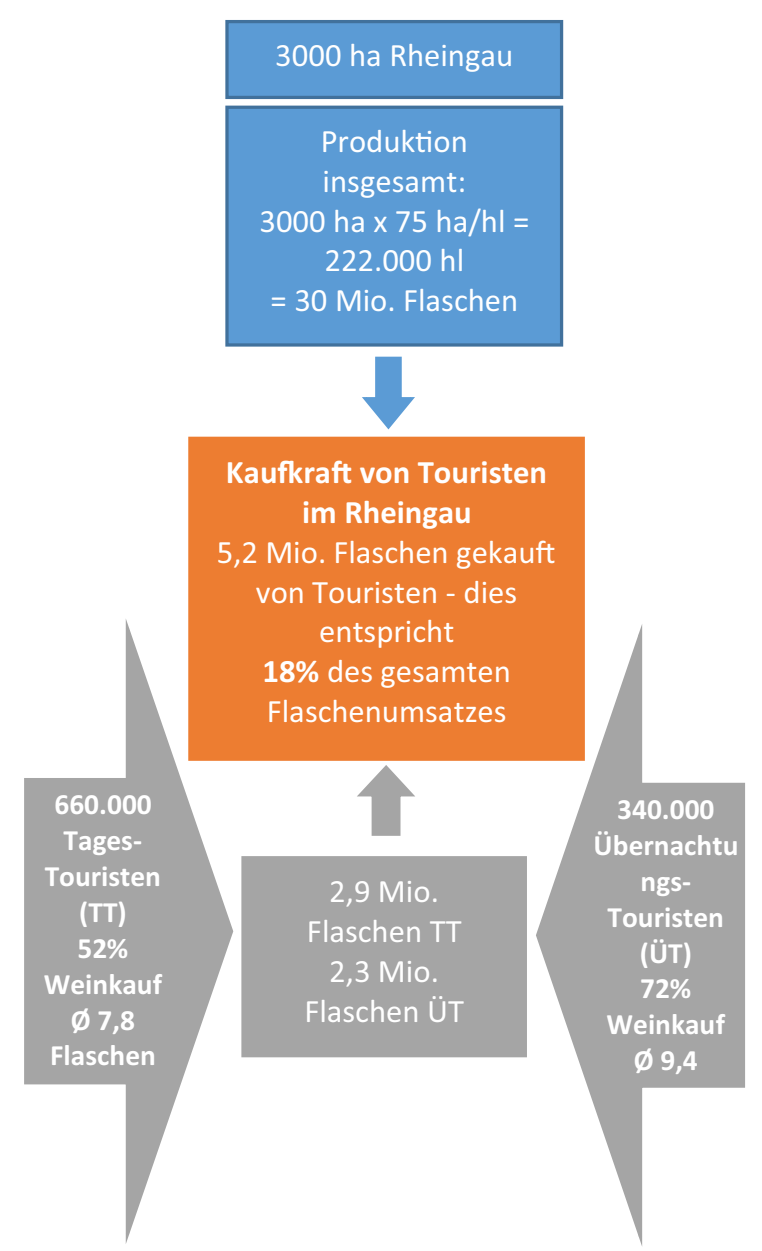

Abb 3. Modellrechnung von Kaufkraft.

dieser Touristengruppe stehen Erholung und Weinreise als Grund an erster Stelle, allerdings spielen auch Kultur- und Bildungsreise eine wichtige Rolle. Als Übernachtungsmöglichkeit wurde hier verstärkt das Hotel genutzt. Die am meisten besuchte Veranstaltung dieses Segments war der Rüdesheimer Weihnachtsmarkt.

\subsection{Kaufkraft von touristen im rheingau}

Speziell die Weinwirtschaft profitiert im Rheingau vom Tourismus und erfährt weiterhin Zulauf. Wie hier eine Modellrechnung zeigt (Abb 3), die mithilfe der gesammelten Daten und öffentlicher Statistiken errechnet werden konnte, ist die Kaufkraft der Besucher im Rheingau nicht $\mathrm{zu}$ unterschätzen und umfasst mit ca. 5 Mio. Flaschen beinahe ein Fünftel des gesamten Flaschenumsatzes.

Der Rheingau hat insgesamt 3.000 ha Rebfläche und wenn mit einem durchschnittlichen Ertrag von $75 \mathrm{hl} /$ ha gerechnet wird, kommt man auf eine Gesamtproduktion von ca. 30 Mio. Flaschen. Laut offizieller Statistiken von Hessisches Statistisches Bundesamt (2015) besuchen die Region jährlich 660.000 Tages- und 340.000 Übernachtungs-Touristen. Aus der Befragung war es $\mathrm{zu}$ entnehmen, dass $52 \%$ der Tages-Touristen und $72 \%$ der Übernachtungs-Touristen im Rheingau Wein kaufen und der Weinanteil 7,8 bzw. 9,4 Flaschen/Kopf beträgt. Auf diese Weise kaufen Touristen in der 
Region insgesamt 5,2 Mio. Flaschen Wein, was 18\% der Gesamtproduktion entspricht.

\section{Fazit}

Der Rheingau ist, verglichen mit anderen deutschen Anbaugebieten relativ klein, hat trotzdem eine große Anzahl an Touristen, welche die Region besuchen. $52 \%$ der Tages-Touristen und $72 \%$ der ÜbernachtungsTouristen kaufen während ihres Aufenthalts regionale Weine ein. Durch die Hochrechnung von Kaufkraft kann es bestätigt werden, dass Tourismus für die Rheingauer Weinwirtschaft ein wichtiges Standbein ist - lokale Produzenten vermarkten $18 \%$ ihrer Weine direkt Ab-Hof an Touristen.

\section{References}

[1] Alebaki, M. \& Iakovidou, O. (2011). Market segmentation in wine tourism: A comparison of approaches. Tourismos, 6(1), 123-140

[2] Alonso, A. D., Fraser, R. A. \& Cohen, D. A. (2007). Investigating differences between domestic and international winery visitors in New Zealand. International Journal of Wine Business Research, 19(2), 114-126

[3] Bitsani, E. \& Kavoura, A. (2012). Connecting oenological and gastronomical tourisms at the wine roads, Veneto, Italy, for the promotion and development of agrotourism. Journal of Vacation Marketing, 18(4), 301-312

[4] Brown, G. P., Havitz, M. E. \& Getz, D. (2006). Relationship between wine involvement and winerelated travel. Journal of Travel and Tourism Marketing, 21(1), 31-46

[5] Bruwer, J. (2003). South African wine routes: Some perspectives on the wine tourism industry's structural dimensions and wine tourism product. Tourism Management, 24(4), 423-435

[6] Charters, S. \& Ali-Knight, J. (2000). Wine tourism-a thirst for knowledge? International Journal of Wine Marketing, 12(3), 70-80

[7] Chen, H-J. \& Sasias, M. (2014). Tourist segmentation in Taiwan's wineries: A cultural perspective. Social Behavior and Personality, 42(2), 223-236

[8] Cho, M., Bonn, M. A. \& Brymer, R. A. (2014). A constraint-based approach to wine tourism market segmentation. Journal of Hospitality and Tourism Research. DOI:10.1177/1096348014538049

[9] Cullen, C.W., Kaciak, E., Bramble, L.,Wright, B., Seaman, A. E. \& Williams, J. J. (2006). Winter wine tourists in Canada's Niagara Region. Proceedings of 3rd International Wine Business Research Conference, July 6-8. 2006, Montpellier, France

[10] Di-Gregorio, D. \& Licari, E. (2006). Rural development and wine tourism in Southern Italy. Proceedings of 46th Congress of the European Regional Science Association, August 30-September 3. 2006, Volos, Greece
[11] Dodd, T. \& Bigotte, V. (1997). Perceptual differences among visitor groups to wineries. Journal of Travel Research, 35(3), 46-51

[12] Galloway, G., Mitchell, R., Getz, D., Crouch, G. \& Ong, B. (2008). Sensation seeking and the prediction of attitudes and behaviours of wine tourists. Tourism Management, 29(5), 950-966

[13] Gatti, S. \&Maroni, F. (2004). A profile of wine tourists in some Italian region vineyards: An application of the multiple correspondence analysis. Proceedings of Vineyard Data Quantification Society (VDQS), May 21-22. 2004, Dijon, France

[14] Hall, C. M. \&Macionis, N. (1998). Wine tourism in Australia and New Zealand, In R.W. Butler, C. M. Hall, \& J. M. Jenkins (Eds.). Tourism and recreation in rural areas (pp. 197-224), Chichester: John Wiley

[15] Hessisches Statistisches Bundesamt (2015). Statistische Berichte - Gäste und Übernachtungen im hessischen Tourismus im Dezember 2014. Wiesbaden: HSB

[16] Houghton, M. (2008). Classifying wine festival customers: Comparing an inductive typology with Hall's wine tourist classification. International Journal of Culture, Tourism and Hospitality Research, 2(1), 67-76

[17] Marzo-Navarro, M., \& Pedraja-Iglesias, M. (2009). Profile of a wine tourist and the correspondence between destination and preferred wine: A study in Aragón, Spain. Journal of Travel and Tourism Marketing, 26(7), 670-687

[18] Molina, A., Gómez, M. González-Díaz, B. \& Esteban, A. (2015). Market segmentation in wine tourism: strategies for wineries and destinations in Spain. Journal of Wine Research. DOI:10.1080/09571264.2015.1051218

[19] Nella, A., \& Christou, E. (2014). Segmenting wine tourists on the basis of involvement with wine. Journal of Travel and Tourism Marketing, 31(7), 783-798

[20] Piscitelli, A., Pomarici, E. \&Tedesco, R. (2005). Wine tourism in Campania region (Italy): Supply potential and demand expectations. Proceedings of International Wine Marketing Symposium, July 8. 2005, Rohnert Park, CA, Sonoma State University, USA

[21] Simpson, K. \& Bretherton, P. (2004). Market segmentation by consumer lifestyle in a wine tourism setting, Proceedings of New Zealand Tourism and Hospitality Research Conference, 8-10. December 2004

[22] Tassiopoulos, D., Nuntsu, N. \& Haydam, N. (2004). Wine tourists in South Africa: A demographic and psychographic study. Journal of Wine Research, 15(1), 51-63

[23] Williams, P. W., \& Kelly, J. (2001). Cultural wine tourists: Product development considerations for British Columbia's resident wine tourism market. International Journal of Wine Marketing, 13(3), 59-76 